\title{
Role of small calibre chest tube drainage for iatrogenic pneumothorax
}

\author{
Michael Laub, Nils Milman, Dorrit Müller, Eivin Struve-Christensen
}

\begin{abstract}
A $2 \mathrm{~mm}$ Teflon catheter was used as a chest tube in 28 patients with iatrogenic pneumothorax. Frequent aspirations through the catheter were performed in 16 of the patients. In the remaining 12 patients the catheter was connected to a one way flutter valve. The treatment was successful in 27 of the 28 patients-one patient required a large calibre chest tube. The mean drainage time was 48 hours. The small catheter technique is superior to the use of a large intercostal drain as it is much less traumatic and troublesome. The small calibre chest tube with a one way valve is recommended as a safe and easy technique.
\end{abstract}

Various methods are available for the management of pneumothorax; most are based on intercostal tube drainage. Smaller tubes ${ }^{1-5}$ and simple aspiration ${ }^{6}$ have been used as alternatives to large intercostal tube drainage. In this paper we examine the efficacy of a small calibre chest tube in the treatment of iatrogenic pneumothorax.

\section{Methods}

During six months 28 consecutive patients, nine women and 19 men aged 28-72 years, had a small calibre chest tube catheter inserted after they developed a pneumothorax complicating percutaneous transthoracic fine needle lung biopsy ( $n=19$ ), fibreoptic transbronchial biopsy $(n=6)$, or pleurocentesis $\mathrm{n}=3$ ). The catheter used was a Secalon $\mathrm{T} 14$ gauge radio-opaque Teflon "over needle" catheter with a FloSwitch stoplock, initially designed for subclavian vein catheterisation (Viggo Products, Swindon, UK; fig 1). The outer diameter was $2 \mathrm{~mm}$ and the inner diameter $1.5 \mathrm{~mm}$. Catheters were inserted for one or more of the following reasons: (1) the apical collapse of the lung was $4 \mathrm{~cm}$ or more; (2) radiographically the pneumothorax had increased in the course of 24 hours; (3) dyspnoea was present. Patients with pleural fluid were excluded because of the risk of obstruction of the catheter or the flutter valve mechanism (see below). The catheter was inserted into the second intercostal space in the midclavicular line after skin disinfection and application of local analgesia. While suction was applied with a $60 \mathrm{ml}$ syringe the catheter was moved over the cranial edge of the third rib in a medial and cephalic direction until air was aspirated. The Teflon catheter was then advanced over the needle and the needle withdrawn. The catheter was secured with adhesive tape. As much air as possible was aspirated by repeated suction with the syringe, and a chest radiograph was obtained to assess any residual pneumothorax.

In 16 patients the FloSwitch was locked after the insertion of the catheter. Aspiration was performed twice daily, or more frequently if the chest radiograph showed a persistent pneumothorax. When no air could be aspirated for 24 hours and the chest radiograph showed no further evidence of the pneumothorax, the catheter was removed. In 12 patients a one way flutter valve (LPP valve, Leo, Copenhagen) (fig 2) was attached to the open FloSwitch and no further aspiration was attempted. When the chest radiograph had shown re-expansion of the lung for 24 hours the FloSwitch was locked. If a chest radiograph taken after another four to six hours was unchanged the catheter was removed.

\section{Results}

One patient in the group in which the catheter was closed developed a large pneumothorax shortly after each aspiration. After 24 hours a large chest tube with underwater drainage was inserted and the patient's air leak stopped

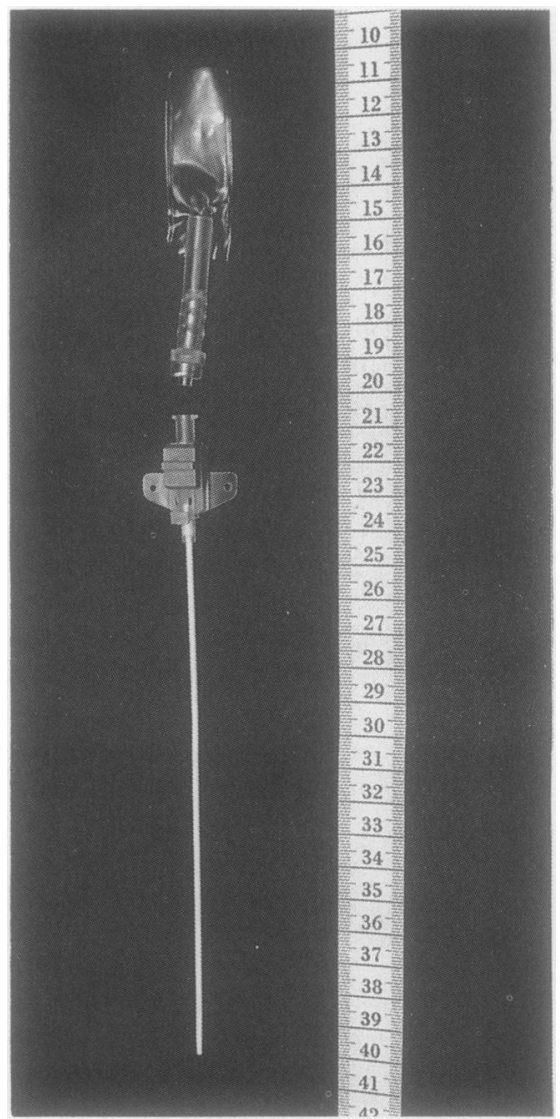

Figure 1 The Secalon $T$ catheter and the LPP one way flutter valve (centimetre scale). The inner needle has been withdrawn from the catheter. 
Figure 2 Close up of the FloSwitch stoplock and the connection to the LPP valve (centimetre scale).

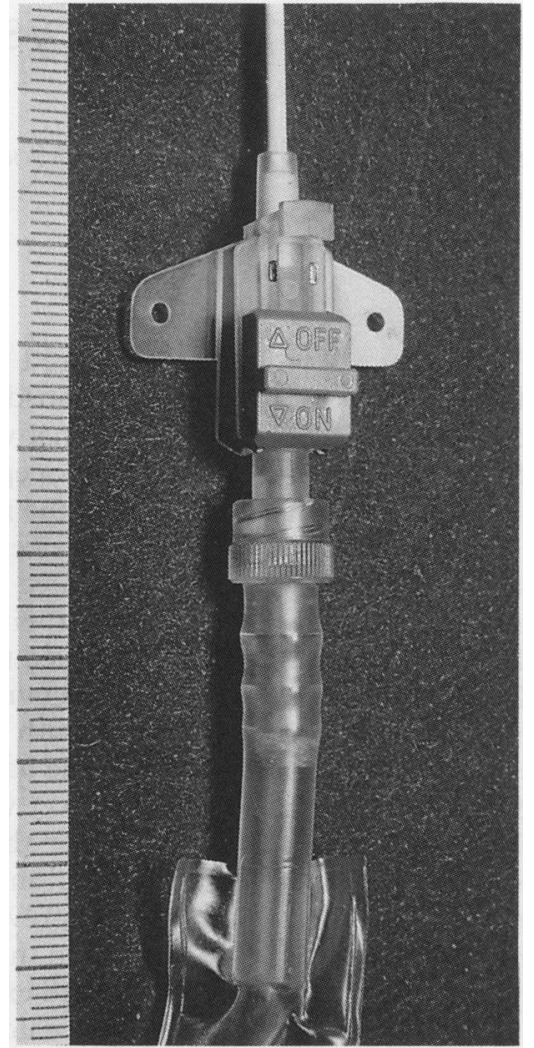

during the third day. In the remaining patients the Secalon catheter provided definitive treatment of the pneumothoraces. In both groups the median drainage time was 48 (range 28-95) hours. Eight patients complained of moderate pain in the upper part of the chest during air aspiration. Eleven patients, seven in the group with the closed catheter and four in the group with the valve, disliked the feeling of the catheter; the rest of the patients had no complaints. No complications relating to the insertion or removal of the catheter were seen.

\section{Discussion}

Of 28 patients who developed a pneumothorax, 27 were treated successfully with a $2 \mathrm{~mm}$ calibre chest catheter. Air was aspirated either by a syringe or passively by a flutter valve. Despite the small difference in the outcome of the two regimens, the one way flutter valve has advantages over the closed catheter technique, both for the patient's safety and for daily use. Firstly, the risk of tension pneumothorax is eliminated only when an open catheter with a flutter valve is used. Secondly, no further syringe aspirations are needed, which is time saving for the physician and more comfortable for the patient.

Hamilton and Archer ${ }^{6}$ and others advocate that smaller spontaneous pneumothoraces can be successfully treated with a single aspiration. If this procedure has to be performed more than once, however, it has no advantages over the small catheter technique. Iatrogenic pneumothorax often develops faster than a spontaneous pneumothorax, and the risk of tension pneumothorax is not eliminated if simple aspiration is done. Furthermore, using a needle instead of a catheter for aspiration carries a risk of lacerating the lung. ${ }^{7}$ Our findings with the until now smallest used chest thoracostomy catheter and those reported by other investigators confirm the efficacy of small catheters for treating pneumothorax. The insertion technique is easy to learn and the possibility of treating a complication of a diagnostic procedure easily results in better patient care and makes a useful diagnostic procedure acceptable to clinicians. Other advantages of the Secalon catheter technique include the rapidity with which the tube is inserted and the good cosmetic result as the small catheter leaves almost no surgical scar.

The Secalon catheter is reserved for patients without pleural fluid (blood, pus, exudation), which could cause the catheter to become occluded. Small amounts of pleural exudation should not, however, be a contraindication, as they can be aspirated through the catheter before the valve is connected.

The traditional underwater drainage method has several disadvantages when compared with the LPP flutter valve. ${ }^{8}$ Some patients are confined to their bed or chair and others move about with difficulty. There is the small risk that the bottle will break, and disposable bottles are expensive. The obvious advantages of the flutter valve are that the patient can walk about, the valve functions in any position, and the mechanism of the valve is easily understood by the medical staff. The valve enables the pleural cavity to be evacuated without the encumbrance of suction, because the expansion of the lung itself forces air out of the chest at the same time that reflux is prevented. The drainage time in our patients was short and did not prolong hospital stay; in contrast, the average hospital stay of patients treated with underwater seal drains is 13 days. $^{910}$ The Secalon catheter with the LPP valve also could be used in patients with iatrogenic pneumothoraces following other procedures, such as central venous catheterisation and intercostal nerve blockage.

In conclusion, treatment of iatrogenic pneumothorax with a small calibre chest tube of $2 \mathrm{~mm}$ diameter connected with a one way flutter valve appears to be a safe and adequate technique, which is less painful and traumatic than large tube thoracostomy.

1 Rohner RC. Zur Behandlung des Spontan und Spannungspneumothorax mittels Plastikvenenkatheters und Saugdrainage. Schweiz Med Wochenschr 1968;98:54-6.

2 Sargent EN, Turner AF. Emergency treatment of pneumothorax: a simple catheter technique for use in the radiology department. AJR 1970;109:531-5.

3 Perlmutt LM, Brain SD, Newman GE, et al. Transthoracic needle aspiration: use of small chest tube to treat pneumothorax. AJR 1987;148:849-51.

4 Conces DJ, Tarver RD, Gray WC, Pearcy EA. Treatment of pneumothoraces utilizing small calibre chest tubes. Chest 1988;94:55-7.

5 Casola G, vanSonnenberg E, Keightley A, Ho M, Withers C, Lee AS. Pneumothorax: radiologic treatment with small catheters. Radiology 1988;166:89-91.

6 Hamilton AAD, Archer GJ. Treatment of pneumothorax by simple aspiration. Thorax 1983;38:934-6.

7 Ruckley CV, McCormack RJM. The management of spontaneous pneumothorax. Thorax 1966;21:139-44.

$8 \mathrm{Heimlich} \mathrm{HJ}$. Valve drainage of the pleural cavity. Dis Chest 1968;53:282-7.

9 Smith WG, Rothwell PPG. Treatment of spontaneous pneumothorax. Thorax 1962;17:342-9.

10 Thompson HT, Bailey RR. Management of spontaneous pneumothorax. NZ Med J 1986;65:101-4. 